Proyecciones Journal of Mathematics

Vol. 35, No 4, pp. 395-404, December 2016.

Universidad Católica del Norte

Antofagasta - Chile

\title{
Energy of strongly connected digraphs whose underlying graph is a cycle
}

\author{
Juan Monsalve \\ Universidad de Antioqua, Colombia \\ and \\ Juan Rada \\ Universidad de Antioquía, Colombia \\ Received : June 2015. Accepted : July 2016
}

\begin{abstract}
The energy of a digraph is defined as $\mathcal{E}(D)=\sum_{k=1}^{n}\left|\operatorname{Re}\left(z_{k}\right)\right|$, where $z_{1}, \ldots, z_{n}$ are the eigenvalues of the adjacency matrix of $D$. This is a generalization of the concept of energy introduced by I. Gutman in 1978 [3]. When the characteristic polynomial of a digraph $D$ is of the form

$$
\phi_{D}(z)=\sum_{k=0}^{\left\lfloor\frac{n}{2}\right\rfloor}(-1)^{k} b_{k}(D) z^{n-2 k}
$$

where $b_{0}(D)=1$ and $b_{k}(D) \geq 0$ for all $k$, we show that

$$
\mathcal{E}(D)=\frac{2}{\pi} \int_{0}^{\infty} \frac{1}{t^{2}} \ln \left[\sum_{k=0}^{\left\lfloor\frac{n}{2}\right\rfloor} b_{k}(D) t^{2 k}\right] d t
$$

This expression for the energy has many applications in the study of extremal values of the energy in special classes of digraphs. In this paper we consider the set $\mathcal{D}^{*}\left(C_{n}\right)$ of all strongly connected digraphs whose underlying graph is the cycle $C_{n}$, and characterize those whose characteristic polynomial is of the form (0.1). As a consequence, we find the extremal values of the energy based on (0.2).
\end{abstract}

Keywords : digraphs; energy; characteristic polynomial; strongly connected; directed cycles.

AMS Subject Classification: 05C35; 05C50; 05 C90. 


\section{Introduction}

A directed graph (or just digraph) $D$ consists of a non-empty finite set $\mathcal{V}$ of elements called vertices and a finite set $\mathcal{A}$ of ordered pairs of distinct vertices called arcs. Two vertices are called adjacent if they are connected by an arc. If there is an arc from vertex $u$ to vertex $v$ we indicate this by writing $u v$. A digraph $D$ is symmetric if $u v \in \mathcal{A}$ then $v u \in \mathcal{A}$, where $u, v \in \mathcal{V}$. A one to one correspondence between graphs and symmetric

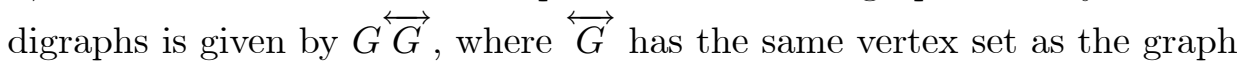
$G$, and each edge $u v$ of $G$ is replaced by a pair of symmetric arcs $u v$ and $v u$. On the other hand, given a digraph $D$ we denote by $\mathcal{U}(D)$ the underlying graph of $D$ defined as the graph with the same set of vertices as $D$, and there is an edge between two vertices $u$ and $v$ of $\mathcal{U}(D)$ if and only if $u v$ or $v u$ is an $\operatorname{arc}$ of $D$.

The adjacency matrix of a digraph $D$ with $n$ vertices $\left\{v_{1}, \ldots, v_{n}\right\}$ is defined as the $n \times n$ matrix $A=\left(a_{i j}\right)$ where

$$
\mathrm{a}_{i j}=\left\{\begin{array}{cc}
1 & \text { if } v_{i} v_{j} \text { is an } \operatorname{arc} \text { of } D \\
0 & \text { if not }
\end{array}\right.
$$

The characteristic polynomial of $D$ is the characteristic polynomial of $A$ and we denoted by $\phi_{D}(z)$. The eigenvalues $z_{1}, \ldots z_{n}$ of $A$ are called the eigenvalues of the digraph $D$. Since $A$ is not necessarily a symmetric matrix, the eigenvalues of $D$ can be complex numbers. The energy of a digraph $D$ is defined as $\mathcal{E}(D)=\sum_{k=1}^{n}\left|\operatorname{Re}\left(z_{k}\right)\right|[6]$, a generalization of the energy of a graph introduced by Gutman in 1978 [3] (see also [4] for more details on this concept and its applications to chemistry). Since Coulson's integral formula holds for the energy of a digraph then

$$
\mathcal{E}(D)=\frac{2}{\pi} \int_{0}^{\infty} \frac{1}{t^{2}} \ln |\gamma(t)| d t
$$

where $\gamma(t)=t^{n} \phi_{D}\left(\frac{i}{t}\right)$ (see ([5] and [6]). When the characteristic polynomial of a digraph $D$ can be expressed as

$$
\phi_{D}(z)=\sum_{k=0}^{\left\lfloor\frac{n}{2}\right\rfloor}(-1)^{k} b_{k}(D) z^{n-2 k}
$$

where $b_{0}(D)=1$ and $b_{k}(D) \geq 0$ for all $k$, we show in Theorem 2.1 that 


$$
\mathrm{E}(D)=\frac{2}{\pi} \int_{0}^{\infty} \frac{1}{t^{2}} \ln \left[\sum_{k=0}^{\left\lfloor\frac{n}{2}\right\rfloor} b_{k}(D) t^{2 k}\right] d t
$$

Consequently, if $D_{1}$ and $D_{2}$ are digraphs with characteristic polynomials expressed as (1.2), then the energy is increasing with respect to the quasiorder relation $D_{1} \preceq D_{2}$ defined as $b_{k}\left(D_{1}\right) \leq b_{k}\left(D_{2}\right)$ for all $k$. This property is essential in order to find extemal values of the energy in special classes of digraphs. So the natural question is: which digraphs satisfy (1.2)?

Let us call $\Omega_{n}$ the set of digraphs with $n$ vertices such that the characteristic polynomial is of the form (1.2). It is well known that the set of bipartite graphs (i.e. bipartite symmetric digraphs) with $n$ vertices is contained in $\Omega_{n}$. This is not true for general bipartite digraphs. For example, if $\overrightarrow{C_{4}}$ is the directed cycle of 4 vertices then $\phi_{\overrightarrow{C_{4}}}(z)=z^{4}-1$ does not alternate signs of the coefficients as in (1.2). It was shown in [7] that the set $\Delta_{n}$ consisting of digraphs with $n$ vertices and such that every cycle has length $\equiv 2(\bmod 4)$ is contained in $\Omega_{n}$. However it is still an open problem to determine exactly which digraphs belong to $\Omega_{n}$.

Our interest in this work is to give some insight in this problem. Specifically, we consider the set $\mathcal{D}^{*}\left(C_{n}\right)$ of strongly connected digraphs whose underlying graph is the cycle $C_{n}$. We first characterize such digraphs and then compute its characteristic polynomial (Lemma 2.2 and Theorem 2.3). From this expression of the characteristic polynomial we characterize the digraphs of $\mathcal{D}^{*}\left(C_{n}\right)$ which belong to $\Omega_{n}$ and then we find the extremal values of the energy (Theorems 2.4 and 2.6).

\section{Energy of strongly connected digraphs whose underlying graph is a cycle}

Let us define a quasi-order relation over $\Omega_{n}$ as follows: if $D_{1}$ and $D_{2}$ have characteristic polynomials

$$
\phi_{D_{i}}(z)=\sum_{k=0}^{\left\lfloor\frac{n}{2}\right\rfloor}(-1)^{k} b_{k}\left(D_{i}\right) z^{n-2 k}
$$

where $b_{0}\left(D_{i}\right)=1$ and $b_{k}\left(D_{i}\right) \geq 0$ for all $k(i=1,2)$, then $D_{1} \preceq D_{2}$ if and only if $b_{k}\left(D_{1}\right) \leq b_{k}\left(D_{2}\right)$ for all $k$. If further $b_{k}\left(D_{1}\right)<b_{k}\left(D_{2}\right)$ for some $k$ then $D_{1} \prec D_{2}$. We first show that the energy is increasing with respect to this quasi-order relation. 
Theorem 2.1. If $D \in \Omega_{n}$ then $E(D)=\frac{2}{\pi} \int_{0}^{\infty} \frac{1}{t^{2}} \ln \left[\sum_{k=0}^{\left\lfloor\left\lfloor\frac{n}{2}\right\rfloor\right.} b_{k}(D) t^{2 k}\right] d t$

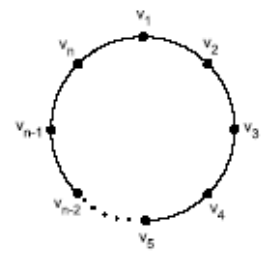

$C_{n}$

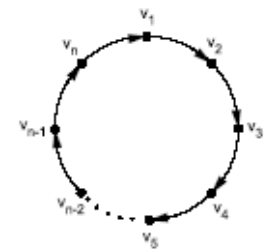

$\overrightarrow{C_{n}}$

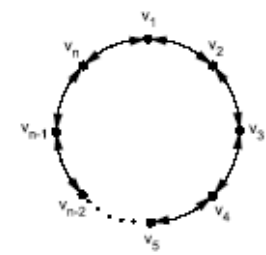

$\overleftrightarrow{C_{n}}$

Figure 1: Cycles and directed cycles

In particular, the energy is increasing over $\Omega_{n}$ with respect to the quasiorder relation $\preceq$.

Proof. From (1.1) $\mathrm{E}(D)=\frac{2}{\pi} \int_{0}^{\infty} \frac{1}{t^{2}} \ln |\gamma(t)| d t$

where $\gamma(t)=t^{n} \phi_{D}\left(\frac{i}{t}\right)$.

Since $D \in \Omega_{n}$ we deduce that

$$
\begin{aligned}
|\gamma(t)| & =\left|t^{n} \phi_{D}\left(\frac{i}{t}\right)\right|=\left|t^{n} \sum_{k=0}^{\left\lfloor\frac{n}{2}\right\rfloor}(-1)^{k} b_{k}(D)\left(\frac{i}{t}\right)^{n-2 k}\right| \\
& =\sum_{k=0}^{\left\lfloor\frac{n}{2}\right\rfloor} b_{k}(D) t^{2 k}
\end{aligned}
$$

and so

$$
\mathrm{E}(D)=\frac{2}{\pi} \int_{0}^{\infty} \frac{1}{t^{2}} \ln |\gamma(t)| d t=\frac{2}{\pi} \int_{0}^{\infty} \frac{1}{t^{2}} \ln \left[\sum_{k=0}^{\left\lfloor\frac{n}{2}\right\rfloor} b_{k}(D) t^{2 k}\right] d t
$$

It follows easily from this expression that the energy is increasing over $\Omega_{n}$ with respect to the quasi-order relation $\preceq$.

As we mentioned in the introduction, the set of symmetric bipartite digraphs with $n$ vertices is contained in $\Omega_{n}$, but for bipartite digraphs in general this is not true. For instance, consider the cycle $C_{n}$ on $n$ vertices, i.e. the vertex of $C_{n}$ is $V\left(C_{n}\right)=\left\{v_{1}, \ldots, v_{n}\right\}$ and the edge set of $C_{n}$ is 
$E\left(C_{n}\right)=\left\{v_{1} v_{2}, v_{2} v_{3}, \ldots, v_{n-1} v_{n}, v_{n} v_{1}\right\}$. Denote by $\overrightarrow{C_{n}}$ the directed cycle and $\overleftrightarrow{C_{n}}$ the symmetric digraph of $C_{n}$ (see Fig. 1). Clearly $\overrightarrow{C_{n}}$ is a strongly connected bipartite digraph when $n$ is even, and its characteristic polynomial is $\phi_{\overrightarrow{C_{n}}}(z)=z^{n}-1$. Then for every $n$ which is a multiple of 4 , $\overrightarrow{C_{n}} \notin \Omega_{n}$.

We will investigate which strongly connected digraphs whose underlying graph is $C_{n}$ belong to $\Omega_{n}$. Let us denote by $\mathcal{D}\left(C_{n}\right)$ the set consisting of all digraphs $D$ such that $\mathcal{U}(D)=C_{n}$. Moreover, we define

$\mathrm{D}^{*}\left(C_{n}\right)=\left\{U \in \mathcal{D}\left(C_{n}\right): U\right.$ is strongly connected $\}$

Lemma 2.2. A digraph $U$ belongs to $\mathcal{D}^{*}\left(C_{n}\right)$ if and only if $U=\overleftrightarrow{C_{n}}$ or $U$ is obtained from $\overleftrightarrow{C_{n}}$ by deleting some arcs of the form $v_{j} v_{j-1}$, where $j=1, \ldots, n\left(v_{0}=v_{n}\right)$.

Proof. Assume that $U \in \mathcal{D}^{*}\left(C_{n}\right)$. Since $\mathcal{U}(D)=C_{n}$, the only possible directed cycles in $U$ are 2-cycles or $\overrightarrow{C_{n}}$. If $U \neq \overleftrightarrow{C_{n}}$ then there exists an arc $u v$ of $U$ such that $v u$ is not an $\operatorname{arc}$ of $U$, in other words, $u v$ does not belong to a 2-cycle. Since $U$ is strongly connected then $\overrightarrow{C_{n}}$ must be a cycle of $U$ (which contains $u v$ ) and so $U$ is obtained from $\overleftrightarrow{C_{n}}$ by deleting some arcs of the form $v_{j} v_{j-1}$, where $j=1, \ldots, n\left(v_{0}=v_{n}\right)$. Conversely, if $Y$ is obtained from $\overleftrightarrow{C_{n}}$ by deleting some arcs of the form $v_{j} v_{j-1}$, then clearly $\mathcal{U}(Y)=C_{n}$ and every arc of $Y$ is contained in the cycle $\overrightarrow{C_{n}}$, so $Y$ is strongly connected.

We next compute the characteristic polynomial of digraphs in $\mathcal{D}^{*}\left(C_{n}\right)$. Given $U \in \mathcal{D}^{*}\left(C_{n}\right)$ and $p$ a positive integer, we will denote by $S_{p}(U)$ the set of $p$ independent 2-cycles of $U$ and $\left|S_{p}(U)\right|$ the number of elements $S_{p}(U)$ has. For instance, for the digraph $Q_{8}$ in Figure 2, $\left|S_{1}\left(Q_{8}\right)\right|=4$, $\left|S_{2}\left(Q_{8}\right)\right|=6,\left|S_{3}\left(Q_{8}\right)\right|=4$ and $\left|S_{4}\left(Q_{8}\right)\right|=1$.

Theorem 2.3. Let $U \in \mathcal{D}^{*}\left(C_{n}\right)$.

1. If $n$ is odd then

$$
\phi_{U}(z)=z^{n}+\sum_{k=1}^{\frac{n-1}{2}}(-1)^{k}\left|S_{k}(U)\right| z^{n-2 k}-1 ;
$$

2. If $n$ is even then 
$\phi_{U}(z)= \begin{cases}z^{n}+\sum_{k=1}^{\frac{n}{2}-1}(-1)^{k}\left|S_{k}(U)\right| z^{n-2 k}-\left(1-\left|S_{\frac{n}{2}}(U)\right|\right) & \text { if } \quad n \equiv 0(\bmod 4) \\ z^{n}+\sum_{k=1}^{\frac{n}{2}-1}(-1)^{k}\left|S_{k}(U)\right| z^{n-2 k}-\left(1+\left|S_{\frac{n}{2}}(U)\right|\right) & \text { if } \quad n \equiv 2(\bmod 4) .\end{cases}$

Proof. Assume that the characteristic polynomial of $U$ is $\phi_{U}(z)=$ $\sum_{k=0}^{n} a_{k} z^{n-k}$, where $a_{0}=1$. Then by Sachs Theorem [2], $a_{j}=\sum_{L \in \mathcal{L}_{j}}(-1)^{p(L)}$ for every $1 \leq j \leq n$, where $\mathcal{L}_{j}$ is the set of linear subdigraphs with $j$ vertices and $p(L)$ is the number of components $L$ has. Since $U \in \mathcal{D}^{*}\left(C_{n}\right)$ has only cycles of length 2 and $\overrightarrow{C_{n}}$, we can compute the linear subdigraphs of $U$ as follows:

1. If $n$ is odd then

$$
\mathcal{L}_{k}(U)=\left\{\begin{array}{ccc}
\emptyset & \text { if } & k \text { is odd, } 1 \leq k \leq n-2 \\
S_{\frac{k}{2}}(U) & \text { if } & k \text { is even, } 2 \leq k \leq n-1 \\
\left\{\overrightarrow{C_{n}}\right\} & \text { if } & k=n
\end{array}\right.
$$

Clearly $a_{j}=0$ for all $j$ odd, $1 \leq j \leq n-2$ and $a_{n}=(-1)^{p\left(\overrightarrow{C_{n}}\right)}=-1$. When $j \equiv 2(\bmod 4)$ then $\frac{j}{2}$ is odd while $j \equiv 0(\bmod 4)$ implies $\frac{j}{2}$ is even. Hence

$$
a_{j}=\sum_{L \in \mathcal{L}_{j}}(-1)^{c(L)}=\left\{\begin{array}{ccc}
-\left|S_{\frac{j}{2}}(U)\right| & \text { if } & j \equiv 2(\bmod 4) \\
\left|S_{\frac{j}{2}}(U)\right| & \text { if } & j \equiv 0(\bmod 4)
\end{array}\right.
$$

and consequently

$$
\phi_{U}(z)=\sum_{k=0}^{\frac{n-1}{2}}(-1)^{k}\left|S_{k}(U)\right| z^{n-2 k}-1
$$

2. If $n$ is even then

$$
\mathcal{L}_{k}(U)=\left\{\begin{array}{ccc}
\emptyset & \text { if } & k=1,3,5, \ldots, n-1 \\
S_{\frac{k}{2}}(U) & \text { if } & k=2,4,6, \ldots, n-2 \\
S_{\frac{n}{2}}(U) \cup\left\{\overrightarrow{C_{n}}\right\} & \text { if } & k=n .
\end{array}\right.
$$

Again $a_{j}=0$ for all $j$ odd, $1 \leq j \leq n-1$. Similarly, when $j$ is even and $2 \leq j \leq n-2$ then $a_{j}$ is given by (2.2). Finally,

$$
\mathrm{a}_{n}=\left\{\begin{array}{c}
-1-\mid S_{\frac{n}{2}}(U) \\
-1+\left|S_{\frac{n}{2}}(U)\right|
\end{array} \mid \begin{array}{cc}
\text { if } & n \equiv 2(\bmod 4)
\end{array}\right. \text { and the result follows. }
$$


Now we can determine the digraphs in $\mathcal{D}^{*}\left(C_{n}\right)$ which belong to $\Omega_{n}$. For $n$ even, let $Q_{n}$ be the digraph obtained from $\overrightarrow{C_{n}}$ by adding the arcs $v_{i} v_{i-1}$ for all $i$ even $(2 \leq i \leq n-2)$ (see Fig. 2). Clearly by Lemma 2.2, $Q_{n} \in \mathcal{D}^{*}\left(C_{n}\right)$ for all positive even integer $n$.

Theorem 2.4. Let $n$ be a positive integer.

1. If $n$ is odd then no digraph in $\mathcal{D}^{*}\left(C_{n}\right)$ belongs to $\Omega_{n}$;

2. If $n \equiv 2(\bmod 4)$ then all digraphs in $\mathcal{D}^{*}\left(C_{n}\right)$ belong to $\Omega_{n}$. Moreover, the cycle $\overrightarrow{C_{n}}$ has the minimal energy and $\overleftrightarrow{C_{n}}$ has the maximal energy over the set $\mathcal{D}^{*}\left(C_{n}\right)$;

3. If $n \equiv 0(\bmod 4)$ then a digraph $U \in \mathcal{D}^{*}\left(C_{n}\right)$ belongs to $\Omega_{n}$ if and only if $U=Q_{n}$ or $U$ is obtained from $Q_{n}$ by adding some arcs of the form $v_{j} v_{j-1}$, where $j=1, \ldots, n\left(v_{0}=v_{n}\right)$. For these digraphs, the minimal energy is attained in $Q_{n}$ and the maximal energy is attained in $\overleftrightarrow{C_{n}}$

Proof. 1. Note that if $n$ is odd and $U \in \mathcal{D}^{*}\left(C_{n}\right) \cap \Omega_{n}$ then $\phi_{U}(0)=0$ and by part 1 of Theorem $2.3, \phi_{U}(0)=-1$, a contradiction. Hence no digraph in $\mathcal{D}^{*}\left(C_{n}\right)$ belongs to $\Omega_{n}$.

2. If $n \equiv 2(\bmod 4)$ and $U \in \mathcal{D}^{*}\left(C_{n}\right)$ then by part 2 of Theorem 2.3

$$
\phi_{U}(z)=z^{n}+\sum_{k=1}^{\frac{n}{2}-1}(-1)^{k}\left|S_{k}(U)\right| z^{n-2 k}-\left(1+\left|S_{\frac{n}{2}}(U)\right|\right)
$$

which clearly satisfies (1.2). Assume that $U \neq \overrightarrow{C_{n}}$. Then by Lemma 2.2, there exists an arc of the form $v_{j} v_{j-1}$ in $U$, for some $j=1, \ldots, n\left(v_{0}=v_{n}\right)$. Let $U^{\prime}$ be the digraph obtained from $U$ by deleting the arc $v_{j} v_{j-1}$. Then clearly $U^{\prime} \in \mathcal{D}^{*}\left(C_{n}\right)$ and $\mathcal{L}_{k}\left(U^{\prime}\right) \subseteq \mathcal{L}_{k}(U)$ for all $k \geq 0$. Hence $\left|S_{k}\left(U^{\prime}\right)\right| \leq$ $\left|S_{k}(U)\right|$ for all $k=1, \ldots, \frac{n}{2}$. In other words, $U^{\prime} \leq U$. Consequently, starting from any digraph $V \in \mathcal{D}^{*}\left(C_{n}\right)$, we can step by step delete an arc of the form $v_{j} v_{j-1}$ to obtain a decreasing sequence of digraphs in $\mathcal{D}^{*}\left(C_{n}\right)$ that ends in $\overrightarrow{C_{n}}$. Similarly, we construct an increasing sequence of digraphs in $\mathcal{D}^{*}\left(C_{n}\right)$ by adding arcs that ends in $\overleftrightarrow{C_{n}}$. Since the energy is increasing with respect to this quasi-order relation by Theorem 2.1 , the result follows. 


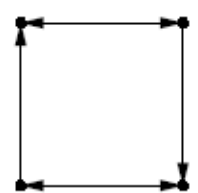

$Q_{4}$

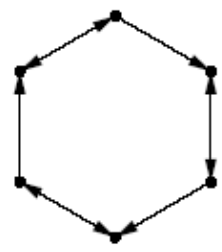

$Q_{6}$

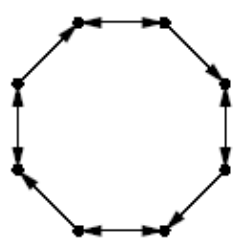

$Q_{8}$

Figure 2: Digraphs in $\mathcal{D}^{*}\left(C_{n}\right) \cap \Omega_{n}$ when $n$ is even

3. If $n \equiv 0(\bmod 4)$ and $W \in \mathcal{D}^{*}\left(C_{n}\right)$ then the characteristic polynomial of $W$ is given by

$$
\phi_{W}(z)=z^{n}+\sum_{k=1}^{\frac{n}{2}-1}(-1)^{k}\left|S_{k}(W)\right| z^{n-2 k}-\left(1-\left|S_{\frac{n}{2}}(W)\right|\right)
$$
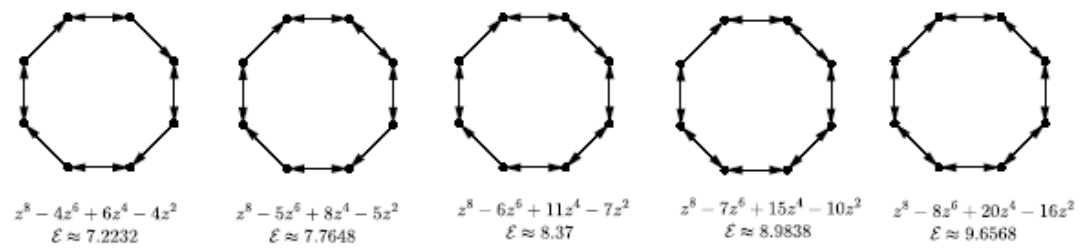

Figure 3: Digraphs in $\Omega_{8} \backslash \Delta_{8}$

Note that when $n \equiv 0(\bmod 4)$ then $\frac{n}{2}-1$ is odd and so $W$ satisfies $(1.2)$ if and only if $-\left(1-\left|S_{\frac{n}{2}}(W)\right|\right) \geq 0$, which is equivalent to $S_{\frac{n}{2}}(W) \neq \emptyset$. But clearly $S_{\frac{n}{2}}(W) \neq \emptyset$ if and only if $W=Q_{n}$ or $W$ is obtained from $Q_{n}$ by adding some arcs of the form $v_{j} v_{j-1}$, where $j=1, \ldots, n\left(v_{0}=v_{n}\right)$. Finally if $Z$ is obtained from $Q_{n}$ by adding some arcs of the form $v_{j} v_{j-1}$, we proceed as in part 2 to delete arcs of the form $v_{j} v_{j-1}$ until reaching $Q_{n}$. Similarly, adding arcs of this form to $Z$ will end in $\overleftrightarrow{C_{n}}$. The result follows again by the increasing property of the energy given in Theorem 2.1.

As we mentioned in the introduction, $\Delta_{n} \subset \Omega_{n}$, where $\Delta_{n}$ is the set of 
digraphs with $n$ vertices and every cycle has length $\equiv 2(\bmod 4)[7]$. Note that Theorem 2.4 gives plenty of examples of digraphs in $\Omega_{n}$ which are not in $\Delta_{n}$.

Example 2.5. $Q_{4 k} \in \Omega_{4 k} \backslash \Delta_{4 k}$ for every $k \geq 1$ and so does every digraph obtained from $Q_{4 k}$ by adding arcs of the form $v_{j} v_{j-1}$, where $j=1, \ldots, 4 k$ $\left(v_{0}=v_{4 k}\right)$. For instance, $Q_{8}$ and the derived digraphs shown in Figure 3.

As we can see in Theorem 2.4, when $n$ is odd no digraph in $\mathcal{D}^{*}\left(C_{n}\right)$ belongs to $\Omega_{n}$. However, in this case we still can find the extremal values of the energy over $\mathcal{D}^{*}\left(C_{n}\right)$.

Theorem 2.6. If $n$ is odd then $\overrightarrow{C_{n}}$ has the minimal energy and $\overleftrightarrow{C_{n}}$ has the maximal energy over the set $\mathcal{D}^{*}\left(C_{n}\right)$.

Proof. Let $U \in \mathcal{D}^{*}\left(C_{n}\right)$. Then by Theorem 2.3

$$
\phi_{U}(z)=z^{n}+\sum_{k=1}^{\frac{n-1}{2}}(-1)^{k}\left|S_{k}(U)\right| z^{n-2 k}-1 ;
$$

and so using directly formula (1.1) we deduce that

$$
\mathcal{E}(U)=\frac{2}{\pi} \int_{0}^{\infty} \frac{1}{t^{2}} \ln |\gamma(t)| d t
$$

where

$$
\begin{gathered}
\quad|\gamma(t)|=\left|t^{n} \phi_{U}\left(\frac{i}{t}\right)\right|=\left|t^{n}\left(\left(\frac{i}{t}\right)^{n}+\sum_{k=1}^{\frac{n-1}{2}}(-1)^{k}\left|S_{k}(U)\right|\left(\frac{i}{t}\right)^{n-2 k}-1\right)\right| \\
=\left|t^{n}-i^{n}\left(1+\sum_{k=1}^{\frac{n-1}{2}}\left|S_{k}(U)\right| t^{2 k}\right)\right| \\
=\sqrt{t^{2 n}+\left(1+\sum_{k=1}^{\frac{n-1}{2}}\left|S_{k}(U)\right| t^{2 k}\right)^{2}}
\end{gathered}
$$

Hence substituting (2.5) in (2.4) we easily deduce that if $U, U^{\prime} \in \mathcal{D}^{*}\left(C_{n}\right)$ are such that $\left|S_{k}(U)\right| \leq\left|S_{k}\left(U^{\prime}\right)\right|$ for all $k=1, \ldots, \frac{n-1}{2}$ then $\mathcal{E}(U) \leq \mathcal{E}\left(U^{\prime}\right)$. In particular, $\overrightarrow{C_{n}}$ has the minimal energy and $\overleftrightarrow{C_{n}}$ has the maximal energy over the set $\mathcal{D}^{*}\left(C_{n}\right)$. 


\title{
References
}

[1] R. Cruz, H. Giraldo, J. Rada, An upper bound for the energy of radial digraphs, Linear Alg. Appl. 442, pp. 75-81, (2014).

[2] D. Cvetković, M. Doob, H. Sachs, Spectra of graphs - Theory and Application, Academic, New York, (1980).

[3] I. Gutman, The energy of a graph. Ber. Math.-Statist. Sekt. Forschungsz. Graz 103, pp. 1-22, (1978).

[4] X. Li, Y. Shi, I. Gutman, Graph energy, Springer-Verlag, New York, (2012).

[5] M. Mateljević, V. Božin, I. Gutman, Energy of a polynomial and the Coulson integral formula. J. Math. Chem. 48, pp. 1062-1068, (2010).

[6] I. Peña, J. Rada, Energy of digraphs, Lin. Multilin. Alg. 56, pp. 565$579,(2008)$.

[7] J. Rada, I. Gutman, R. Cruz, The energy of directed hexagonal systems, Linear Alg. Appl. 439, pp. 1825-1833, (2013).

\author{
Juan Monsalve \\ Instituto de Matemáticas, \\ Universidad de Antioquia \\ Medellín, \\ Colombia \\ e-mail : jdmonsal@gmail.com \\ and

\section{Juan Rada} \\ Instituto de Matemáticas, \\ Universidad de Antioquia \\ Medellín, \\ Colombia \\ e-mail : pablo.rada@udea.edu.co
}

